\title{
Estimation of the diaphragm neuromuscular efficiency index in mechanically ventilated critically ill patients
}

Diana Jansen', Annemijn H. Jonkman², Lisanne Roesthuis³, Suvarna Gadgil ${ }^{4}$, Johannes G. van der Hoeven³, Gert-Jan J. Scheffer ${ }^{1}$, Armand Girbes ${ }^{2}$, Jonne Doorduin ${ }^{5}$, Christer S. Sinderby ${ }^{6}$ and Leo M. A. Heunks²*

\begin{abstract}
Background: Diaphragm dysfunction develops frequently in ventilated intensive care unit (ICU) patients. Both disuse atrophy (ventilator over-assist) and high respiratory muscle effort (ventilator under-assist) seem to be involved. A strong rationale exists to monitor diaphragm effort and titrate support to maintain respiratory muscle activity within physiological limits. Diaphragm electromyography is used to quantify breathing effort and has been correlated with transdiaphragmatic pressure and esophageal pressure. The neuromuscular efficiency index (NME) can be used to estimate inspiratory effort, however its repeatability has not been investigated yet. Our goal is to evaluate NME repeatability during an end-expiratory occlusion (NMEoccl) and its use to estimate the pressure generated by the inspiratory muscles (Pmus).

Methods: This is a prospective cohort study, performed in a medical-surgical ICU. A total of 31 adult patients were included, all ventilated in neurally adjusted ventilator assist (NAVA) mode with an electrical activity of the diaphragm (EAdi) catheter in situ. At four time points within $72 \mathrm{~h}$ five repeated end-expiratory occlusion maneuvers were performed. NMEocd was calculated by delta airway pressure $(\Delta$ Paw)/ $\Delta$ EAdi and was used to estimate Pmus. The repeatability coefficient $(R C)$ was calculated to investigate the NMEoccl variability.

Results: A total number of 459 maneuvers were obtained. At time $T=0$ mean NMEoccl was $1.22 \pm 0.86$ $\mathrm{cmH}_{2} \mathrm{O} / \mu \mathrm{V}$ with a RC of $82.6 \%$. This implies that when NMEoccl is $1.22 \mathrm{cmH}_{2} \mathrm{O} / \mu \mathrm{V}$, it is expected with a probability of $95 \%$ that the subsequent measured NMEoccl will be between 2.22 and $0.22 \mathrm{cmH} 2 \mathrm{O} / \mu \mathrm{V}$. Additional EAdi waveform analysis to correct for non-physiological appearing waveforms, did not improve NMEoccl variability. Selecting three out of five occlusions with the lowest variability reduced the RC to $29.8 \%$.

Conclusions: Repeated measurements of NMEoccl exhibit high variability, limiting the ability of a single NMEoccl maneuver to estimate neuromuscular efficiency and therefore the pressure generated by the inspiratory muscles based on EAdi.
\end{abstract}

Keywords: Diaphragm dysfunction, Neuromuscular efficiency index, Mechanical ventilation, Partially supported mode, Diaphragm electromyography, Monitoring

\footnotetext{
*Correspondence: l.heunks@vumc.nl

${ }^{2}$ Department of Intensive Care Medicine, Amsterdam UMC, Vrije Universiteit

Amsterdam, Postbox 7057, 1007, MB, Amsterdam, The Netherlands

Full list of author information is available at the end of the article
}

(c) The Author(s). 2018 Open Access This article is distributed under the terms of the Creative Commons Attribution 4.0 International License (http://creativecommons.org/licenses/by/4.0/), which permits unrestricted use, distribution, and reproduction in any medium, provided you give appropriate credit to the original author(s) and the source, provide a link to the Creative Commons license, and indicate if changes were made. The Creative Commons Public Domain Dedication waiver (http://creativecommons.org/publicdomain/zero/1.0/) applies to the data made available in this article, unless otherwise stated. 


\section{Background}

Diaphragm dysfunction frequently develops in mechanically ventilated intensive care unit (ICU) patients and is associated with adverse clinical outcomes including prolonged mechanical ventilation and mortality [1-7]. It appears that non-physiological diaphragm activity plays an important role [8], in which both disuse atrophy resulting from ventilator over-assist $[3,9,10]$ and high respiratory muscle effort resulting from ventilator under-assist [1113] have been associated with diaphragm dysfunction in ICU patients. Therefore, there is a strong physiological rationale for monitoring diaphragm effort [14-16] and titrating support to maintain respiratory muscle activity within physiological limits [17].

Variations in esophageal pressure (Pes) during breathing have been used for decades to quantify breathing effort. Recently, two state-of-the-art papers reviewed the technical and clinical aspects of esophageal pressure (Pes) monitoring in ICU patients [18, 19]. Limitations of this technique include strict control of balloon inflation volume and complexity of signal interpretation, in particular when expiratory muscles are recruited.

Diaphragm electromyography (EMG) is an alternative technique used to quantify breathing effort in ICU patients [15]. Strong correlation has been reported between the electrical activity of the diaphragm (EAdi) and transdiaphragmatic pressure (Pdi) or Pes [20, 21]. The neuromechanical efficiency (NMEoccl), defined by delta airway pressure $(\Delta \mathrm{Paw})$ divided by $\Delta \mathrm{EAdi}$ measured during an end-expiratory occlusion, has been used to estimate the inspiratory effort breath by breath [21, 22]. This ratio describes how much pressure can be generated for each microvolt of EAdi signal, in other words how efficient the diaphragm is in generating pressure for a certain amount of electrical activity. This is of potential interest for monitoring diaphragm function (over time) and helps to titrate ventilatory support in order to minimize diaphragm dysfunction resulting from ventilator over-assist and under-assist [17]. Today, this index has only been evaluated in studies including limited numbers of patients [21-24] and the repeatability, an essential characteristic for a diagnostic tool, has not been investigated at all. Therefore, the aim of our study was to evaluate the NMEoccl repeatability in mechanically ventilated ICU patients and its use to estimate the maximum inspiratory pressure generated by the inspiratory muscles (Pmus).

\section{Methods}

\section{Study design and population}

This prospective cohort study was performed in an academic ICU. Adult patients, with a dedicated EAdi catheter (Maquet critical care, Solna Sweden) in situ and mechanically ventilated in neurally adjusted ventilator assist (NAVA) mode were eligible for inclusion. The institutional ethical committee approved the study protocol and informed consent was waived due to the non-invasive nature of the study and negligible risks.

\section{Study protocol}

The EAdi catheter was positioned according to the manufacturer's instructions using a dedicated software tool on the Servo-i ventilator. The catheter position was verified before data acquisition. When patients exhibited a stable breathing pattern (i.e. no coughing, hiccups or disproportional differences in respiratory rate), measurements to assess respiratory muscle function were performed: (1) an end-expiratory occlusion maneuver (obtained by activating the expiratory hold button on the ventilator for one inspiratory effort) for measurement of NMEoccl and (2) a "zero assist breath" in which inspiratory support was decreased to zero for one single breath to calculate patient-ventilator breath contribution (PVBC) $[25,26]$. Both maneuvers were repeated five times with at least a one-minute interval. The number of breaths between maneuvers was variable to prevent anticipation by the patient. Measurements were recorded and stored for offline analysis at time $T=0$ and after $12 \mathrm{~h}(T=12), 24 \mathrm{~h}(T=24)$ and $72 \mathrm{~h}(T=72)$.

\section{Data acquisition}

EAdi, flow and Paw waveforms were acquired from the Servo-i ventilator via a RS232 serial port connected to a laptop with dedicated software (Servo Tracker version 4.1, Maquet, Solna, Sweden). Maximum inspiratory pressure (MIP) measurements were performed with a manovacumeter (Micro Respiratory Pressure Meter, Carefusion, Yorba Linda, CA, USA) connected to the endotracheal tube [27].

\section{Data analysis}

A software routine developed for MatLab (version R2016b, MathWorks Inc., Natick, MA, USA) was used for offline analysis.

NMEoccl was calculated in three different ways (Fig. 1): (1) by dividing $\triangle$ Paw by $\Delta \mathrm{EAdi}[21]$, in which $\Delta$ Paw is the difference in pressure between the lowest Paw during end-expiratory occlusion and the preceding endexpiratory pressure level, (2) by dividing the area under the curve (AUC) of Paw and EAdi, and (3) by dividing Paw and EAdi at fixed points (steps of $3 \mu \mathrm{V}$ ) on the EAdi waveform. The inspiratory time $(\mathrm{Ti})$ was defined as the period between the onset of EAdi and 70\% peak of EAdi. To investigate the effect of an occlusion on the inspiratory time, we compared $\mathrm{Ti}$ during occlusion with the $\mathrm{Ti}$ of three preceding unloaded breaths. To estimate Pmus under clinical conditions, the mean EAdi of five tidal breaths (before the end-expiratory occlusion) was 


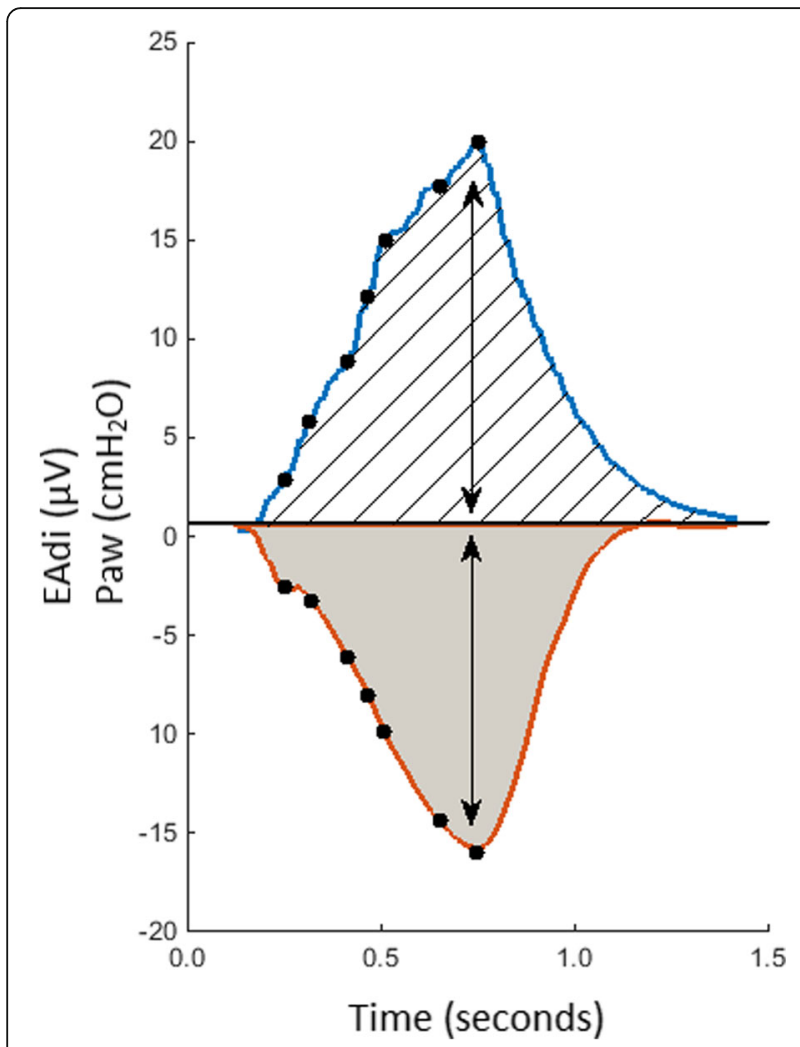

Fig. 1 Example of a single neuromechanical efficiency index during an end-expiratory occlusion (NMEoccl) maneuver. The blue line represents the electrical activity of the diaphragm (EAdi) signal expressed in microvolts. The orange line represents the airway pressure (Paw) expressed in centimeters of water. As described above, the NMEoccl was calculated in three different ways, with the calculation based on (1) delta peak values of electrical activity of the diaphragm (EAdi) and Paw, shown as arrows; (2) area under the curve (AUC) of the EAdi and Paw signal, shown by diagonal lines and gray area, respectively; (3) using fixed points (steps of $3 \mu \mathrm{V}$ ) on the EAdi curve (during inspiration) and corresponding Paw, shown as black dots

multiplied by NMEoccl/1.5 [21]. The correction of 1.5 compensates for the fact that in the presence of flow, the diaphragm generates less pressure for the same EAdi than during an occlusion [21]. The tension-time index (TTI) was calculated as Pmus/MIP multiplied by the ratio of $\mathrm{Ti}$ to total respiratory cycle time $\left(\mathrm{T}_{\text {tot }}\right)[28]$.

\section{Statistical analysis}

Statistical analysis was performed with GraphPad PRISM (version 5.03 for Mac/Windows, Software Inc. San Diego, CA, USA). Data were analyzed as median \pm interquartile range (IQR), except as stated otherwise. Statistical significance was indicated by a $p$ value $<0.05$.

The repeatability coefficient $(\mathrm{RC})$ represents the absolute value by which two repeated measurements in one subject will differ in $95 \%$ of cases. The formula developed by Bland and Altman was used to calculated RC:
$1.96 \times \sqrt{ } 2 \times$ Within-subject standard deviation (SD) [29]. One-way analysis of variance (ANOVA) was used to obtain the within-subject SD with the subject as dependent factor and the repeated NMEoccl measurements as independent factors. Since the NMEoccl variability increased as the magnitude of NMEoccl increased, the ratio of a single NMEoccl measurement to the mean NMEoccl of five repeated measurements was used (see Additional file 1) [29]. The correlation coefficient with repeated observation was used to investigate the within-subject correlation between Paw and EAdi [30, 31] (IBM SPSS Statistics version 22).

In addition, to obtain the within-subject NMEoccl variation, per patient for each time point a coefficient of variation $(\mathrm{CoV})$ was calculated by the ratio of the within-subject SD to the mean. The median $\mathrm{CoV}$ was used to divide the study population into two groups with $\mathrm{CoV}$ higher or lower than median. The paired $t$ test was used to test for differences in clinical parameters (that might affect NMEoccl variability) between both groups.

The Pearson correlation coefficient was calculated to test correlation between $\mathrm{Ti}$ and NMEoccl, and between TTI and Pmus. One-way ANOVA was used to investigate the changes in NMEoccl and inspiratory muscle pressure over time.

\section{Results}

Table 1 shows the main characteristics of the study population. A total 459 occlusions were performed (see Additional file 2). In 19 patients, the measurements could not be obtained at all four time points due to various reasons: extubation $(n=7)$, agitation $(n=2)$, low EAdi $(n=3)$, return to controlled mode $(\mathrm{n}=2)$, death $(n$ $=1)$ and others $(n=4)$.

\section{NMEoccl variability}

A representative NMEoccl maneuver is shown in Fig. 1. At $T=0,149$ maneuvers were obtained in 31 patients, 6 maneuvers were lost due to technical issues. Mean $\Delta$ Paw was $14.1 \pm 7.9 \mathrm{cmH}_{2} \mathrm{O}$ and mean $\Delta$ EAdi 14.8 $\pm 9.9 \mu \mathrm{V}$. Mean NMEoccl was $1.22 \pm 0.86 \mathrm{cmH}_{2} \mathrm{O} / \mu \mathrm{V}$ (ranging from 0.41 to $3.56 \mathrm{cmH}_{2} \mathrm{O} / \mu \mathrm{V}$ ), with a $\mathrm{RC}$ of $82.6 \%$. This implies that when NMEoccl is $1.0 \mathrm{cmH}_{2} \mathrm{O} /$ $\mu \mathrm{V}$, it is expected with a probability of $95 \%$ that the subsequent measured NMEoccl will be between 0.17 and $1.83 \mathrm{cmH}_{2} \mathrm{O} / \mu \mathrm{V}$. When the EAdi of this patient during normal breathing is $10 \mu \mathrm{V}$, the estimated pressure generated by the inspiratory muscles would be somewhere between $1.1 \mathrm{cmH}_{2} \mathrm{O}$ and $12.2 \mathrm{cmH}_{2} \mathrm{O}$ (calculated as EAdi * $\mathrm{NMEoccl} / 1.5$, see "Methods"). In addition, the RC of NMEoccl calculated by the AUC and at fixed points on the EAdi curve (Fig. 2) remained high: $87.7 \%$ for AUC and $85.5-175.9 \%$ at fixed points. The RC of the separate components of NMEoccl was $95.7 \%$ for EAdi and $73.9 \%$ 
Table 1 Main characteristics of the study population

\begin{tabular}{|c|c|}
\hline Characteristics & $N=31$ \\
\hline Age (years), median [IQR] & $69[55.5-72]$ \\
\hline Sex, male/female & $22 / 9$ \\
\hline BMI $\left(\mathrm{kg} / \mathrm{m}^{2}\right)$, median $[\mathrm{QQR}]$ & $24.7[21.6-26]$ \\
\hline \multicolumn{2}{|l|}{ Comorbidity, $n(\%)$} \\
\hline Cardiac diseases & $9(29 \%)$ \\
\hline Diabetes mellitus & $6(19 \%)$ \\
\hline COPD & $4(13 \%)$ \\
\hline \multicolumn{2}{|l|}{ Reason for admission, $n(\%)$} \\
\hline Pneumonia & $13(42 \%)$ \\
\hline Postoperative & $8(26 \%)$ \\
\hline Trauma & $7(23 \%)$ \\
\hline Others & $3(10 \%)$ \\
\hline ARDS at admission, $n(\%)$ & $9(29 \%)$ \\
\hline Sepsis during admission, $n$ (\%) & $6(19 \%)$ \\
\hline Duration of MV on $T=0$ (days), median [IQR] & $10[8.5-18.5]$ \\
\hline Partially supported mode before $T=0$ & $9[4-14]$ \\
\hline Controlled mode before $T=0$ & $1[0-3.5]$ \\
\hline Total days of MV (days), median [IQR] & $24[14.5-29.5]$ \\
\hline NAVA level, median [IQR] & $0.7[0.5-1.2]$ \\
\hline Tidal volume $(\mathrm{ml})$, median $[\mathrm{IQR}]$ & $450[381-554]$ \\
\hline Respiratory rate (per minute), median [IQR] & 25 [18-30] \\
\hline PEEP $\left(\mathrm{cmH}_{2} \mathrm{O}\right)$, median [IQR] & $8[6-10]$ \\
\hline Use of opioids/sedatives, $n$ (\%) & $16(51.6 \%)$ \\
\hline Total LOS ICU (days), median [IQR] & $26[20-34]$ \\
\hline Total LOS hospital (days), median [IQR] & $41[23-52.5]$ \\
\hline Died within the study period, $n(\%)$ & $1(3 \%)$ \\
\hline
\end{tabular}

Abbreviations: ARDS acute respiratory distress syndrome, $B M I$ body mass index, COPD chronic obstructive pulmonary disease, ICU intensive care unit, IQR interquartile range, LOS length of stay, MV mechanical ventilation, NAVA neurally adjusted ventilatory assist, PEEP positive end-expiratory pressure

for Paw. Figure 2 shows there was moderate correlation between $\triangle \mathrm{EAdi}$ and $\triangle \mathrm{Paw}$ in the individual patient (mean $r=0.52, p<0.0005$; ranging from $r=-0.90$ to $r=1.0$ ).

Upon visual inspection it appeared that some of the EAdi tracings exhibit a rather non-physiological shape: a plateau during inspiration (while an increase would be expected) or at maximum inspiration, or a delayed increase in EAdi relative to the decrease in Paw (Fig. 3). It was reasoned that non-physiological-appearing EAdi waveforms contribute to the high NMEoccl variability. Additional file 2 shows every occlusion maneuver obtained in this study. Different mathematical approaches were used in order to try to objectively detect and exclude non-physiological EAdi waveforms (See Additional file 3). Despite these mathematical approaches the NMEoccl repeatability remained high with a RC of $63.4 \%$.

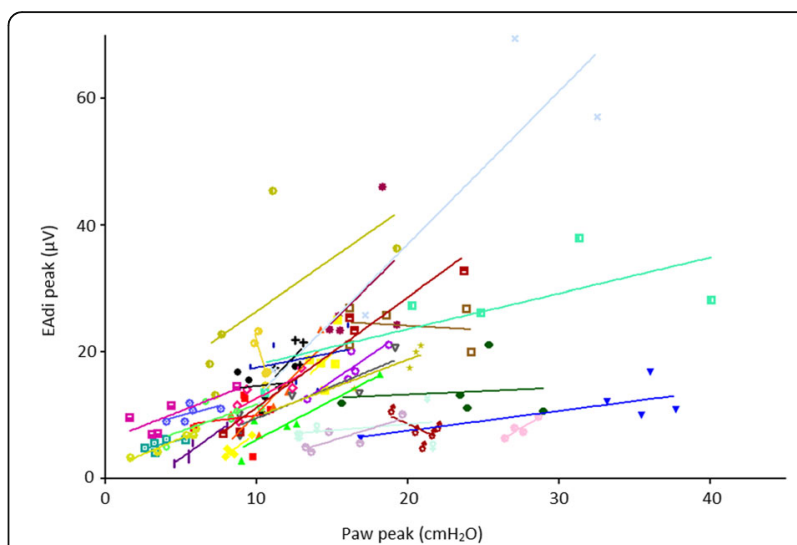

Fig. 2 Overview of the correlation of airway pressure (Paw) peak and electrical activity of the diaphragm (EAdi) peak of all maneuvers at time $T=0$. Each color represents an individual patient with five repeated measurements (dots) and the corresponding slope (line)

Finally, we pragmatically selected three out of five occlusions with the lowest variability and averaged the three values to obtain a single NMEoccl for each individual patient. This approach reduces the influence of erroneous values, irrespective of the origin, and will result in a more reproducible NMEoccl value. As a result, the RC improved to $29.8 \%$. This approach was used for subsequent analysis.

\section{Correlation of NMEoccl variability with clinical parameters and inspiratory time}

The median $\mathrm{CoV}$ NMEoccl at $T=0$ was $23.1 \%$ (IQR 18.7-29.9\%). After dividing the study population into two groups with CoV higher or lower than median, respectively, no significant differences in clinical parameters were found (see Additional file 4). Median Ti during occlusion was $0.51 \mathrm{~s}$ (IQR $0.35-0.65 \mathrm{~s}$ ) and of the preceding breaths $0.65 \mathrm{~s}$ (IQR $0.48-0.84 \mathrm{~s}$ ). On average, in $48 \%$ of the measurements within a patient, Ti of the occluded breath was longer compared to $\mathrm{Ti}$ of the preceding breaths. Only moderate negative correlation was found between Ti and NMEoccl $(r=-0.219)$.

\section{Changes in NMEoccl over time}

Twelve patients completed the $72 \mathrm{~h}$ study period. In these patients, NMEoccl at $T=0$ was $0.8 \mathrm{cmH}_{2} \mathrm{O} / \mu \mathrm{V}$ (IQR 0.7-1.1 $\mathrm{cmH}_{2} \mathrm{O} / \mu \mathrm{V}$ ) and did not change over time $(p=0.75) ; 0.7 \mathrm{cmH}_{2} \mathrm{O} / \mu \mathrm{V}$ (IQR 0.4-0.9 $\left.\mathrm{cmH}_{2} \mathrm{O} / \mu \mathrm{V}\right), 0.8$ $\mathrm{cmH}_{2} \mathrm{O} / \mu \mathrm{V}$ (IQR 0.6-1.1 $\mathrm{cmH}_{2} \mathrm{O} / \mu \mathrm{V}$ ), $0.9 \mathrm{cmH}_{2} \mathrm{O} / \mu \mathrm{V}$ (IQR $0.6-1.3 \mathrm{cmH}_{2} \mathrm{O} / \mu \mathrm{V}$ ) for $T=12, T=24$ and $T=72$, respectively.

\section{Diaphragm muscle effort}

The mean EAdi of five unloaded breaths before the end-expiratory occlusion were used to calculate Pmus. At $T=0$, median Pmus was 9.4 $\mathrm{cmH}_{2} \mathrm{O}$ (IQR 6.0-12.8 


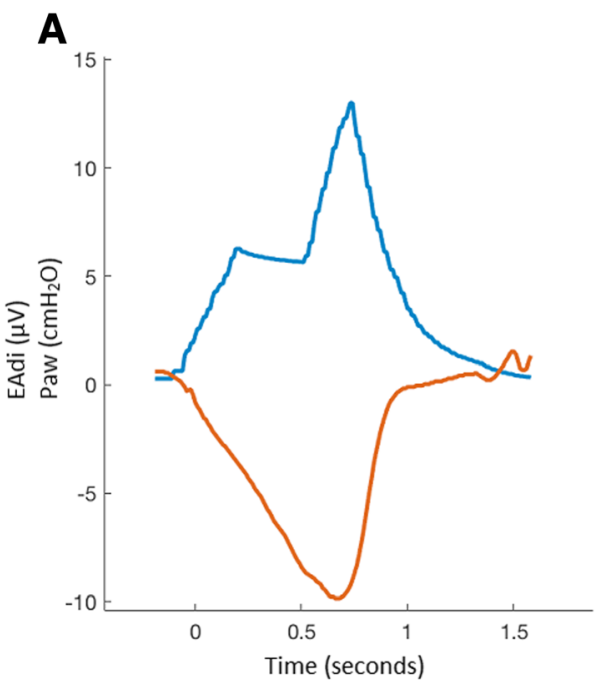

\section{B}
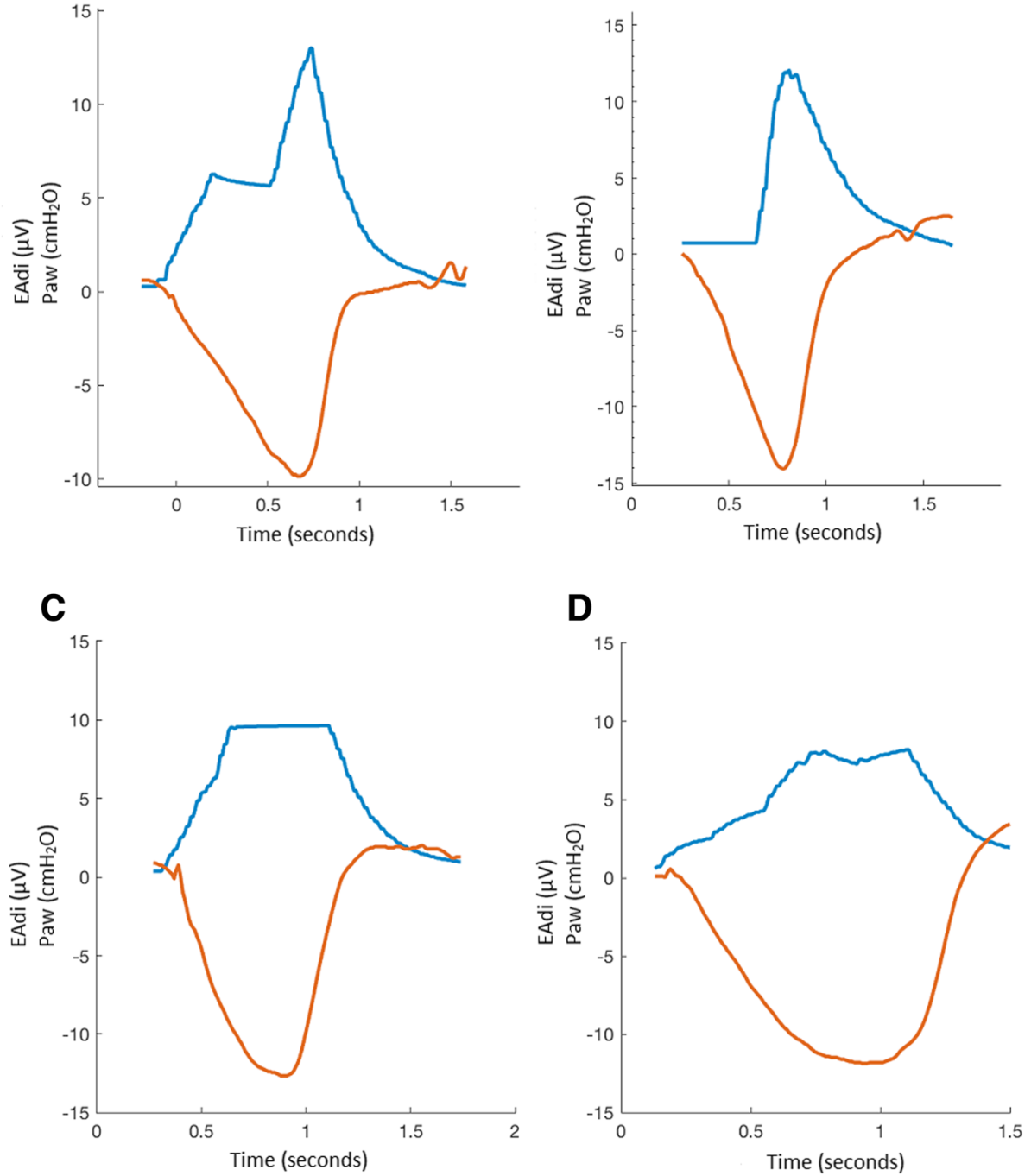

Fig. 3 Four examples of electrical activity of the diaphragm (EAdi) waveform irregularities during an end-expiratory occlusion. The blue line represents the EAdi signal expressed in microvolts. The orange line represent the airway pressure (Paw) expressed in centimeters of water. a Slope $<0$ during the ascending part of the EAdi waveform. b Delay in start of EAdi peak. $\mathbf{c}$ EAdi peak cut off. $\mathbf{d}$ Split EAdi peak

$\left.\mathrm{cmH}_{2} \mathrm{O}\right)$ and did not change over time $(p=0.58) ; 10.4$

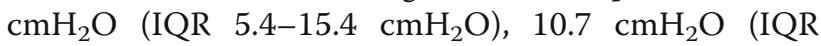
4.8-13.1 $\mathrm{cmH}_{2} \mathrm{O}$ ) and $8.2 \quad \mathrm{cmH}_{2} \mathrm{O}$ (IQR 6.0-15.9 $\mathrm{cmH}_{2} \mathrm{O}$ ) for $T=12, \mathrm{~T}=24$ and $T=72$, respectively. The median Pmus varied widely among patients, ranging from 1.8 to $36.0 \mathrm{cmH}_{2} \mathrm{O}$. Based on previous literature [21, 23], we defined a physiological Pmus between 5 and $10 \mathrm{cmH}_{2} \mathrm{O}$. At $T=0,6$ patients (19.4\%) had a mean Pmus $<5 \mathrm{cmH}_{2} \mathrm{O}$ and 12 patients $(38.7 \%)>10 \mathrm{cmH}_{2} \mathrm{O}$, indicating ventilator over-assist or under-assist, respectively.

MIP was obtained in 15 patients in the week before or after the NME measurements, which allowed us to calculate TTI. Median MIP was $29 \mathrm{cmH}_{2} \mathrm{O}$ (IQR 24$38 \mathrm{cmH}_{2} \mathrm{O}$ ) and median TTI was 0.12 (IQR $0.08-$
0.17). Figure 4 shows the relationship between TTI and Pmus.

\section{Discussion}

The main findings of the present study can be summarized as follows: (1) repeated measurements of NMEoccl within an individual patient exhibited unacceptably high variation, indicating that a single NMEoccl cannot be used to estimate pressure generated by the inspiratory muscles; (2) no correlation was found between NMEoccl variability and clinical parameters; (3) extensive waveform analyses did not improve the repeatability of NMEoccl; (4) NMEoccl and Pmus remain stable over time in a heterogeneous group of patients and (5) both 


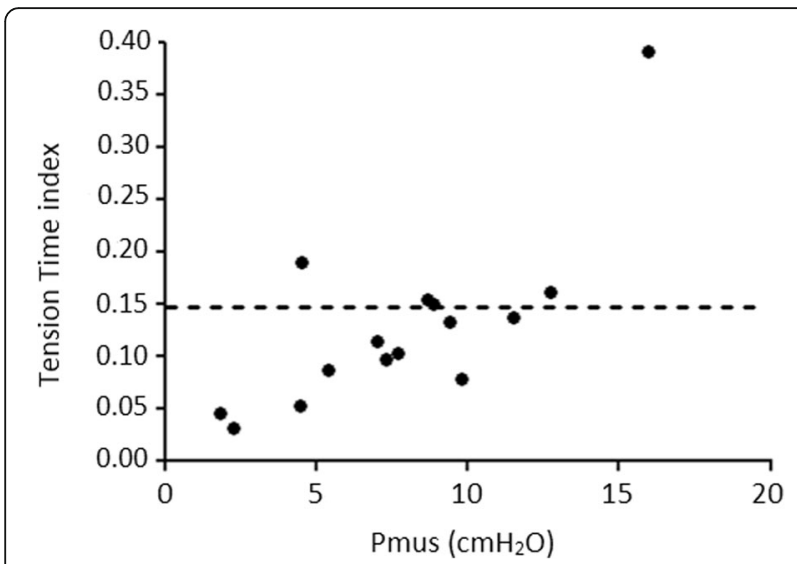

Fig. 4 Overview of correlation between the tension-time index and inspiratory pressure (Pmus) in 15 patients in whom maximum inspiratory pressure was measured (dots). The dotted line represents the cut off for diaphragm fatigue [36]

low and high diaphragm effort are common in this cohort of patients on partially supported mode.

\section{NMEoccl variability}

Variability of both components of NMEoccl (Paw and EAdi) is expected given the variability in inspiratory drive, even during occlusions. However, NMEoccl itself is independent from respiratory drive and should therefore be more stable from breath to breath.

Beck and colleagues reported a linear relationship between EAdi and Pdi in healthy subjects, at least up to $75 \%$ of maximum force [32]. Their subsequent study also demonstrated a linear relationship between EAdi and Pdi in patients with acute respiratory failure [20]. Bellani et al. showed that NMEoccl derived from airway pressure closely reflects NME during normal breathing (NMEdyn) derived from Pes and concluded that calculation of NMEoccl allows a clinically valuable estimate of inspiratory effort [21]. In this latter study, two end-expiratory occlusions were obtained in each patient, but repeatability was not reported. Furthermore, they report that despite changes in level of support, NMEoccl remained rather stable within individual patients, as supported by the linear relationship between Pmus and EAdi $\left(r^{2}=0.78\right)$ [21] This is in apparent contrast with our findings, as we found only moderate correlation between Pmus and EAdi (Fig. 2). However, differences in data analysis should be acknowledged. Bellani used the Pearson correlation coefficient to test the correlation between Paw and EAdi, in which all measurements from different patients were analyzed together as if they were from a single patient. However, the variability of between-subject measurements is different compared to the variability of the within-subject measurements [33]. Therefore, calculating the correlation coefficient of repeated observations, as in the current study, seems more appropriate [30, 31]. Indeed, Fig. 2 demonstrates that the slope of Paw and EAdi in the individual patient is highly variable. This is consistent with the results of Bellani, exhibiting high interindividual variability [21].

The high NMEoccl repeatability reported in the current study precludes its application in clinical practice. Based on visual inspection of the EAdi waveforms, we proposed that suboptimal filtering and replacement of cardiac electric activity by the ventilator software are important. Several techniques for waveform analyses were applied, but did not improve repeatability, suggesting that in addition to suboptimal filtering other factors might be involved. Beck et al. showed that an increase in volume from functional reserve capacity (FRC) to total lung capacity (TLC) reduces Pdi by $60 \%$ for a given EAdi [34]. Similarly, muscle weakness may affect NMEoccl, but both are unlikely to explain the high variability in our study, given that all measurements were obtained in a time window of $5-10 \mathrm{~min}$.

As none of the techniques for waveform analyses resulted in improved NMEoccl repeatability, a more pragmatic approach was explored that is also feasible in clinical practice. In thermodilution cardiac output measurements, three repeated measurements are averaged, provided that these values are within $10 \%$ of their average. If not, a total of five measurements are performed in which the lowest and highest value are eliminated [35]. In our study, this strategy reduced the influence of erroneous NMEoccl values, irrespective of the cause, and will result in a more reproducible value of NMEoccl.

\section{NMEoccl as monitoring tool}

Theoretically, the NMEoccl could be helpful to titrate ventilator support in patients on partially supported modes. NMEoccl is calculated by dividing $\triangle \mathrm{Paw}$ by $\triangle$ EAdi during an end-expiratory occlusion. During an occlusion $\Delta$ Paw equals $\Delta$ Pmus and therefore NMEoccl can be obtained without direct measurement of Pmus (requiring an esophageal balloon). Rearranging this formula to Pmus $=$ NMEoccl * EAdi allows calculation of Pmus breath by breath, after dividing this value by 1.5 to correct for differences in NME obtained under static and dynamic conditions [21]. Interestingly, NMEoccl may be used to evaluate respiratory muscle function over time. A decrease in NMEoccl indicates that the respiratory muscles are less efficient in converting electrical activity into pressure. Possible causes for variability in NMEoccl require further studies, but may include intrinsic positive end-expiratory pressure (PEEP) and impaired function of the contractile proteins.

In our study population there were no significant changes in NMEoccl over time $(p=0.75)$, which corresponds to the 
results of Bellani et al. [23]. However, it should be noted that during our study period the ventilatory settings were not fixed, which might explain why NMEoccl did not change over time.

In our study Pmus varied among patients but remained relatively stable in individual patients over time. In some patients estimated inspiratory effort was $>20 \mathrm{cmH}_{2} \mathrm{O}$. An important question is whether a relatively high inspiratory pressure generated by the respiratory muscles may result in the development of contractile fatigue. A TTI $\geq 0.15$ puts the diaphragm at risk of development of fatigue [36]. In our study, all patients except for two, with a Pmus $<12 \mathrm{cmH}_{2} \mathrm{O}$, had a TTI $<0.15$. This might suggest that titration of ventilatory support to a pressure $<12 \mathrm{cmH}_{2} \mathrm{O}$ could limit the risk of fatigue development. However, this has to be studied before it can be applied in clinical practice.

\section{Strengths and limitations}

The strengths of our study are the high number of occlusions analyzed and the fact that at each time point five repeated occlusions were obtained. This allows thorough analysis of the repeatability of NMEoccl and provides methods to improve its variability under clinical conditions. In addition, several waveform analysis techniques were performed to evaluate the high NMEoccl variability; however, this did not improve the $\mathrm{RC}$ for NMEoccl. It was suggested that suboptimal filtering might be important. Software engineers should further improve ventilator software for EAdi signal filtering.

Several limitations should be acknowledged. First, patients in our study did not have an esophageal balloon in situ and therefore we could not validate our measurements against the gold standard. However, previous studies have shown excellent correlation between $\Delta \mathrm{Paw}$ and $\Delta$ Pes during an occlusion maneuver [37-39]. Second, our study was conducted in a single center and in a selected group of ICU patients. The generalizability of the findings needs to be assessed.

\section{Conclusion}

End-expiratory occlusion allows measurement of static change in EAdi and Paw for calculation of NMEoccl. This maneuver is simple to conduct and safe in ICU patients ventilated in partially supported mode. However, the present study demonstrates that a single maneuver cannot be used to calculate NMEoccl, given the unacceptably high variability. Further studies should be conducted to improve software for EAdi analysis for this specific purpose. For now, selecting three out of five occlusions with the lowest variability seems to be the best method to estimate inspiratory muscle effort from EAdi.

\section{Additional files}

Additional file 1: NMEocd calculated as a ratio. Since the variability of NMEoccl increased as the magnitude of the NMEoccl increased, the ratio of a single NMEoccl value to the mean NMEoccl of five repeated measurements was used to calculate the variability of NMEoccl [26]. (A) The difference in NMEoccl is expressed against mean NMEoccl. (B) the ratio of NMEoccl is expressed against mean NMEoccl. (TIF $70 \mathrm{~kb}$ )

Additional file 2: Overview of the five repeated occlusions in each individual patient. The blue line represents the EAdi signal expressed in microvolts. The orange line represents the airway pressure (Paw) expressed in $\mathrm{CmH}_{2} \mathrm{O}$. (DOCX $1942 \mathrm{~kb}$ )

Additional file 3: Extensive waveform analyses. This file gives an overview of the extensive waveform analyses that are performed, in order to objectively detect and exclude non-physiological EAdi waveforms. (DOCX $36 \mathrm{~kb}$ )

Additional file 4: Correlation of clinical parameters and NMEoccl variability at $T=0$. First, the coefficient of variation (CoV) was calculated for each patient. The median CoV for NMEoccl at $T=0$ was $23.1 \%$ (IQR 18.7-29.9\%). The study population was divided in two groups, with the CoV higher or lower than the median, respectively. $\mathrm{CoV}=$ coefficient of variation; $\mathrm{COPD}=$ chronic obstructive pulmonary disease; $\mathrm{EAdi}=$ electrical activity of the diaphragm; $H R$ = heart rate; $I Q R$ = interquartile range; $\mathrm{NAVA}=$ neutrally adjusted ventilatory assist; RASS $=$ Richmond agitation sedation scale; $\mathrm{RR}=$ respiratory rate; $\mathrm{VT}=$ tidal volume. (DOCX $18 \mathrm{~kb}$ )

\section{Abbreviations \\ ANOVA: Analysis of variance; AUC: Area under the curve; CoV: Coefficient of variation; EAdi: Electrical activity of the diaphragm; EMG: Electromyography; FRC: Functional reserve capacity; ICU: Intensive Care Unit; IQR: Interquartile range; MIP: Maximum inspiratory pressure; NMEdyn: Neuromechanical efficiency index during normal breathing; NMEoccl: Neuromechanical efficiency index (during an end-expiratory occlusion); Paw: Airway pressure; Pdi: Transdiaphragmatic pressure; Pes: Esophageal pressure; Pmus: Inspiratory pressure; PVBC: Patient ventilator breath contribution; RC: Repeatability coefficient; SD: Standard deviation; Ti: Inspiratory time; TLC: Total lung capacity; TTl: Tension-time index; Ttot: Total respiratory cycle time; $\Delta$ : Delta}

\section{Acknowledgements}

For technical help: Norman Comtois, St. Michael's Hospital, University of Toronto, ON, Canada.

\section{Funding}

This study was investigator initiated and financed by institutional resources of the Radboud university medical center, Nijmegen, the Netherlands.

\section{Availability of data and materials}

All data generated during this study are included in this published article, found in the Supplemental Data File.

\section{Authors' contributions}

Study conception and design: DJ, SG, LR, JD, LMAH. Acquisition of data: DJ, LR, SG, LMAH. Analysis and interpretation: DJ, LR, AHJ, JD, CSS, LMAH. Drafting of manuscript: DJ, AHJ, LMAH. Critical revision: AHJ, JGvdH, GJS, ARJG, JD, CSS, $\mathrm{LMAH}$. All authors read and approved the final manuscript.

\section{Ethics approval and consent to participate}

Institutional ethical committee (CMO Region Arnhem - Nijmegen) approved the study protocol (case number 2015-1799, file code kYMYB) and informed consent was waived due to the non-invasive nature of the study and negligible risks.

Consent for publication

Not applicable.

Competing interests

The authors declare that they have no competing interests. 


\section{Publisher's Note}

Springer Nature remains neutral with regard to jurisdictional claims in published maps and institutional affiliations.

\section{Author details}

'Department of Anesthesiology, Radboud University Medical Center, Nijmegen, The Netherlands. ${ }^{2}$ Department of Intensive Care Medicine, Amsterdam UMC, Vrije Universiteit Amsterdam, Postbox 7057, 1007, MB, Amsterdam, The Netherlands. ${ }^{3}$ Department of Intensive Care Medicine, Radboud University Medical Center, Nijmegen, The Netherlands. ${ }^{4}$ Department of Anesthesiology, University Medical Center Utrecht, Utrecht, The Netherlands. ${ }^{5}$ Department of Neurology, Donders Institute, Radboud University Medical Center, Nijmegen, The Netherlands. ${ }^{6}$ Department of Critical Care Medicine, St. Michael's Hospital, University of Toronto, Toronto, ON, Canada.

\section{Received: 7 May 2018 Accepted: 28 August 2018}

Published online: 27 September 2018

\section{References}

1. Dres M, Dube BP, Mayaux J, Delemazure J, Reuter D, Brochard L, et al. Coexistence and impact of limb muscle and diaphragm weakness at time of liberation from mechanical ventilation in medical intensive care unit patients. Am J Respir Crit Care Med. 2017;195(1):57-66.

2. Demoule A, Jung B, Prodanovic H, Molinari N, Chanques G, Coirault C, et al. Diaphragm dysfunction on admission to the intensive care unit. Prevalence, risk factors, and prognostic impact-a prospective study. Am J Respir Crit Care Med. 2013;188(2):213-9.

3. Jaber S, Petrof BJ, Jung B, Chanques G, Berthet JP, Rabuel C, et al. Rapidly progressive diaphragmatic weakness and injury during mechanical ventilation in humans. Am J Respir Crit Care Med. 2011;183(3):364-71.

4. Hermans G, Agten A, Testelmans D, Decramer M, Gayan-Ramirez G. Increased duration of mechanical ventilation is associated with decreased diaphragmatic force: a prospective observational study. Crit Care. 2010;14(4):R127.

5. Jaber S, Jung B, Matecki S, Petrof BJ. Clinical review: ventilator-induced diaphragmatic dysfunction-human studies confirm animal model findings! Crit Care. 2011;15(2):206.

6. Supinski GS, Callahan LA. Diaphragm weakness in mechanically ventilated critically ill patients. Crit Care. 2013;17(3):R120.

7. Goligher EC, Dres M, Fan E, Rubenfeld GD, Scales DC, Herridge MS, et al. Mechanical ventilation-induced diaphragm atrophy strongly impacts clinical outcomes. Am J Respir Crit Care Med. 2017;197(2):204-13.

8. Dres M, Goligher EC, Heunks LMA, Brochard L. Critical illness-associated diaphragm weakness. Intensive Care Med. 2017;43(10):1441-52.

9. Levine S, Nguyen T, Taylor N, Friscia ME, Budak MT, Rothenberg P, et al. Rapid disuse atrophy of diaphragm fibers in mechanically ventilated humans. N Engl J Med. 2008:358(13):1327-35.

10. Goligher EC, Fan E, Herridge MS, Murray A, Vorona S, Brace D, et al. Evolution of diaphragm thickness during mechanical ventilation. Impact of inspiratory effort. Am J Respir Crit Care Med. 2015;192(9):1080-8.

11. Ebihara S, Hussain SN, Danialou G, Cho WK, Gottfried SB, Petrof BJ. Mechanical ventilation protects against diaphragm injury in sepsis: interaction of oxidative and mechanical stresses. Am J Respir Crit Care Med. 2002;165(2):221-8

12. Reid WD, Huang J, Bryson S, Walker DC, Belcastro AN. Diaphragm injury and myofibrillar structure induced by resistive loading. J Appl Physiol (1985). 1994;76(1):176-84.

13. Orozco-Levi M, Lloreta J, Minguella J, Serrano S, Broquetas JM, Gea J. Injury of the human diaphragm associated with exertion and chronic obstructive pulmonary disease. Am J Respir Crit Care Med. 2001;164(9):1734-9.

14. Heunks LM, Doorduin J, van der Hoeven JG. Monitoring and preventing diaphragm injury. Curr Opin Crit Care. 2015;21(1):34-41.

15. Doorduin J, van Hees HW, van der Hoeven JG, Heunks LM. Monitoring of the respiratory muscles in the critically ill. Am J Respir Crit Care Med. 2013; 187(1):20-7.

16. Jonkman AH, Jansen D, Heunks LM. Novel insights in ICU-acquired respiratory muscle dysfunction: implications for clinical care. Crit Care. 2017;21(1):64

17. Heunks L, Ottenheijm C. Diaphragm-protective mechanical ventilation to improve outcomes in ICU patients? Am J Respir Crit Care Med. 2018;197(2): $150-2$.
18. Mauri T, Yoshida T, Bellani G, Goligher EC, Carteaux G, Rittayamai N, et al. Esophageal and transpulmonary pressure in the clinical setting: meaning, usefulness and perspectives. Intensive Care Med. 2016;42(9):1360-73.

19. Akoumianaki E, Maggiore SM, Valenza F, Bellani G, Jubran A, Loring SH, et al. The application of esophageal pressure measurement in patients with respiratory failure. Am J Respir Crit Care Med. 2014;189(5):520-31.

20. Beck J, Gottfried SB, Navalesi P, Skrobik Y, Comtois N, Rossini M, et al. Electrical activity of the diaphragm during pressure support ventilation in acute respiratory failure. Am J Respir Crit Care Med. 2001;164(3):419-24.

21. Bellani G, Mauri T, Coppadoro A, Grasselli G, Patroniti N, Spadaro S, et al. Estimation of patient's inspiratory effort from the electrical activity of the diaphragm. Crit Care Med. 2013;41(6):1483-91.

22. Liu L, Liu H, Yang Y, Huang Y, Liu S, Beck J, et al. Neuroventilatory efficiency and extubation readiness in critically ill patients. Crit Care. 2012;16(4):R143.

23. Bellani G, Coppadoro A, Pozzi M, Bronco A, Albiero D, Eronia N, et al. The ratio of inspiratory pressure over electrical activity of the diaphragm remains stable during ICU stay and is not related to clinical outcome. Respir Care. 2016;61(4):495-501.

24. Di Mussi R, Spadaro S, Mirabella L, Volta CA, Serio G, Staffieri F, et al. Impact of prolonged assisted ventilation on diaphragmatic efficiency: NAVA versus PSV. Crit Care. 2016;20:1

25. Grasselli G, Beck J, Mirabella L, Pesenti A, Slutsky AS, Sinderby C. Assessment of patient-ventilator breath contribution during neurally adjusted ventilatory assist. Intensive Care Med. 2012;38(7):1224-32.

26. Liu L, Liu S, Xie J, Yang Y, Slutsky AS, Beck J, et al. Assessment of patientventilator breath contribution during neurally adjusted ventilatory assist in patients with acute respiratory failure. Crit Care. 2015;19:43.

27. Grams ST, Kimoto KY, Azevedo EM, Lanca M, Albuquerque AL, Brito CM, et al. Unidirectional expiratory valve method to assess maximal inspiratory pressure in individuals without artificial airway. PLoS One. 2015;10(9): e0137825.

28. Vassilakopoulos T, Zakynthinos S, Roussos C. The tension-time index and the frequency/tidal volume ratio are the major pathophysiologic determinants of weaning failure and success. Am J Respir Crit Care Med. 1998;158(2):378-85.

29. Bland JM, Altman DG. Measuring agreement in method comparison studies. Stat Methods Med Res. 1999:8(2):135-60.

30. Bland JM, Altman DG. Calculating correlation coefficients with repeated observations: part 1--correlation within subjects. BMJ. 1995;310(6977):446.

31. Bland JM, Altman DG. Calculating correlation coefficients with repeated observations: part 2--correlation between subjects. BMJ. 1995;310(6980):633.

32. Beck J, Sinderby $C$, Lindstrom $L$, Grassino A. Crural diaphragm activation during dynamic contractions at various inspiratory flow rates. J Appl Physiol (1985). 1998;85(2):451-8

33. Bland JM, Altman DG. Correlation, regression. and repeated data. BMJ. 1994; 308(6933):896

34. Beck J, Sinderby C, Lindstrom L, Grassino A. Effects of lung volume on diaphragm EMG signal strength during voluntary contractions. J Appl Physiol (1985). 1998;85(3):1123-34.

35. Medin DL, Brown DT, Wesley R, Cunnion RE, Ognibene FP. Validation of continuous thermodilution cardiac output in critically ill patients with analysis of systematic errors. J Crit Care. 1998;13(4):184-9.

36. Bellemare F, Grassino A. Effect of pressure and timing of contraction on human diaphragm fatigue. J Appl Physiol Respir Environ Exerc Physiol. 1982; 53(5):1190-5.

37. Watson AC, Hughes PD, Louise Harris M, Hart N, Ware RJ, Wendon J, et al. Measurement of twitch transdiaphragmatic, esophageal, and endotracheal tube pressure with bilateral anterolateral magnetic phrenic nerve stimulation in patients in the intensive care unit. Crit Care Med. 2001;29(7): 1325-31.

38. Buscher H, Valta P, Boie T, Hinz J, Moerer O, Sydow M, et al. Assessment of diaphragmatic function with cervical magnetic stimulation in critically ill patients. Anaesth Intensive Care. 2005;33(4):483-91.

39. Baydur A, Cha EJ, Sassoon CS. Validation of esophageal balloon technique at different lung volumes and postures. J Appl Physiol (1985). 1987;62(1): 315-21. 